\title{
Channel Aware Cooperative FEC/ARQ Mechanism based on Kalman Filter Prediction for Wireless Sensor Networks
}

\author{
http://dx.doi.org/10.3991/ijoe.v10i1.3164 \\ Yong Jin ${ }^{1}$, Kaijian Xia ${ }^{2}$ \\ ${ }^{1}$ Changshu Institute of Technology, Changshu 215500, China \\ ${ }^{2}$ Changshu No.1 People's Hospital
}

\begin{abstract}
The QoS-Guarantee of data delivery over wireless link is a big challenge due to the unreliable and dynamic characteristics of wireless sensor networks. In this paper, a cooperative forward error correction (FEC)/automatic repeat request (ARQ) mechanism was proposed, in the hope that the data packets could be received on receiver node with high quality. The mechanism, based on Kalman filter, predicts the bit error rate and adjusts the channel state parameters according to the bit error rate threshold value. Both the mathematical analyses and NS simulation demonstrate that the proposed mechanism achieves better performance, in terms of throughput, delay, reliability and energy efficiency comparing with traditional FEC/ARQ, cooperative FEC and cooperative ARQ.
\end{abstract}

Index Terms-Wireless sensor networks, Cooperative communication, Relay selection, Kalman filter, Forward Error Correction (FEC), Automatic Repeat reQuest (ARQ).

\section{INTRODUCTION}

Transmission over wireless channel suffers from the limited bandwidth, high packet loss rate, dynamic channel state, sensor nodes mobility, etc $[1,2]$. The above characteristics bring about significant challenges and design considerations for providing QoS guarantees of various applications in complicated wireless sensor networks. Furthermore, different application service inherently has different QoS requirements [3] in terms of throughput, real time performance and reliability, etc. Specially, it is a big challenge how to take advantage of the channel state information to discover the optimal relay nodes for forwarding data. Above all, relay selection protocols and error control schemes play a major part in the framework of wireless sensor networks, so that the application's diversity requirements could be satisfied efficiently.

The QoS guarantee based on cooperative communication in wireless sensor networks has been fairly well studied in the literature [4]-[12]. The main idea of literature [4] is that a hybrid FEC/ARQ mechanism is introduced to improve the reliability and real-time performance for wireless media streaming. M. Tacca et al. analyzed the use of cooperative ARQ protocols in energy harvesting sensor networks, which enables sensor nodes to save their energy consumption in order to heighten the battery recharge rate [5]. In the Forward Error Correction (FEC) approach proposed in literature [6], the redundancy degree of FEC and size of transport packet are adjusted simultaneously in accordance with a minimum bandwidth consumption strategy. Literature [7] discussed the energy efficiency of FEC on distance and predicts the frame loss rate with GM $(1,1)$ model, on the basis of adjusting the FEC parameter of sensor nodes. M. Mardani et al. proposed a cooperative ARQ based on link adaptive and QoS provisioning, as well as research of application to relay assisted and mobile satellite communication [8].

In $[9,10]$, the energy efficiency analysis method was adopted. The difference is that first one is for vehicular Ad hoc networks and the other is for cooperative networks. In [11], an analytical framework was proposed for determining the outage probability of random and best relay selection scheme, which ensures a target QoS, as well as reduces the signaling overhead and the relay selection delay. An output threshold multiple relay selection schemes in cooperative wireless networks is proposed in [12], which provides more flexibility in utilizing bandwidth and spatial diversity.

However, there are some drawbacks in all these methods and research findings explained above. The influence of the information from the dynamic channel state on the quality of cooperative transmission is not concerned in $[5,6]$. The channel state information such as packet error rate, bit error rate, etc is not mentioned in [4, 8]. The reliability and real-time performance of cooperative transmission is not mentioned in [3], their focus is on energy efficiency analysis. How to allocate bandwidth dynamically and set up the parameters of error control schemes adaptively was not considered in $[11,12]$.

In this paper, we analyze the cooperative FEC/ARQ mechanism, in the context of wireless sensor networks. We use analytical and simulation approaches to compare and investigate packet error rate, average delay, throughput ratio and energy efficiency of different error control schemes. The results show that our proposed mechanism is capable of utilizing the available network resource and achieve good perceptual quality, in terms of system throughput, reliability and real-time performance, as well as maximization lifetime of wireless sensor networks.

\section{KALMAN FILTER PREDICTION ALGORITHM}

\section{A. Bit error rate prediction method}

A prediction method based on Kalman filter [13, 14] is introduced to predict bit error rate of the wireless 
channel. On this basis, we design a channel-aware relay selection mechanism. The aim is to improve the reliability and real time performance of wireless communication. In this work, we make use of the shot transition information with Kalman filter, to predict the bit error rate in a short period of time.

First of all, the state vector of state model of Kalman filter are defined, which is used to predict a transmission bit sequence size as the formula (1).

$$
s(t)=\left[\begin{array}{c}
q(t) \\
q^{\prime}(t) \\
q^{\prime \prime}(t)
\end{array}\right]
$$

Where, $q(t)$ means size of sending bits in $t$ time, and $q^{\prime}(t)$ means the velocity that is the first derivative about time of $q(t)$. And $q^{\prime \prime}(t)$ means the accelerated velocity that is the second derivative about time of $q(t)$.

Similarly, the state vector of predicting the size of lost bits is defined as formula (2). Here, $p(t)$ means size of lost or damaged bits in time $t$, and $p^{\prime}(t)$ means the velocity that is the first variation about time of $q(t)$. In addition, $p^{\prime \prime}(t)$ means the accelerated velocity that is the second variation about time of $q(t)$.

$$
s L(t)=\left[\begin{array}{l}
p(t) \\
p^{\prime}(t) \\
p^{\prime \prime}(t)
\end{array}\right]
$$

Another important factor, the state transition matrix is composed of a state model, which can be defined as formula (3).

$$
\Phi(\Delta t)=\left(\begin{array}{ccc}
1 & \Delta t & \frac{\Delta t^{2}}{2} \\
0 & 1 & \Delta t \\
0 & 0 & 1
\end{array}\right)
$$

Where, $\Delta t$ is the measure time interval.

According to the state transition matrix, the state vector of system transit with a variation of time. Therefore, size of sending bits in $t$ time calculates as reflecting a velocity and accelerated velocity in size of bits in $t-\Delta t$ time. A variation of bits size in $t$ time calculates using a velocity and accelerated velocity in $t-\Delta t$ time. That is, the results that multiplied state transition matrix by state vector are expressed to formula (4) and (5).

$$
\begin{aligned}
& q(t)=q(t-\Delta t)+q^{\prime}(t-\Delta t) \cdot \Delta t+q^{\prime \prime}(t-\Delta t) \cdot \Delta t^{2} \\
& q^{\prime}(t)=q^{\prime}(t-\Delta t)+q^{\prime \prime}(t-\Delta t) \cdot \Delta t \\
& q^{\prime \prime}(t)=q^{\prime \prime}(t-\Delta t) \\
& p^{\prime}(t)=p(t-\Delta t)+p^{\prime}(t-\Delta t) \cdot \Delta t+p^{\prime \prime}(t-\Delta t) \cdot \Delta t^{2} \\
& p^{\prime}(t)=p^{\prime}(t-\Delta t)+p^{\prime \prime}(t-\Delta t) \cdot \Delta t \\
& p^{\prime \prime}(t)=p^{\prime \prime}(t-\Delta t)
\end{aligned}
$$

The measurement vector should be designed, which is used to design the measurement model. Actually, this vector is a matrix, which means that measurement is possible among state vector of system from the outside. Size of sending bits and lost bits prove to be a factor of measurement vector. Therefore, measurement vector is defined by formula (6) and (7).

$$
\begin{aligned}
& z(t)=(q) \\
& z L(t)=(p)
\end{aligned}
$$

Observation matrix is used to extract a measurement vector from state vector. Therefore, observation matrix is defined as formula (8) by structure of the state vector and measurement vector.

$$
H(t)=(1,0,0)
$$

There are three phases in the prediction algorithm based on Kalman filter, which are initialization, state prediction, and measurement update. The structure of Kalman filter is set and initialized in initialization step. In state prediction step, state vector forecasted in $t$ time from state vector updated in $t-\Delta t$ time predicts through state transition as formula (9), (10), (11) and (12).

$$
\begin{gathered}
\hat{s}^{-}(t)=\Phi(\Delta t) \hat{s}(t-\Delta t) \\
P^{-}(t)=\Phi(\Delta t) \cdot P(t-\Delta t) \Phi(\Delta t)^{t} \\
s L^{\wedge^{-}}(t)=\Phi(\Delta t) s L^{\wedge}(t-\Delta t) \\
P L^{-}(t)=\Phi(\Delta t) \cdot P L(t-\Delta t) \Phi(\Delta t)^{t}
\end{gathered}
$$

The system state is updated after calculating an error between actual measured vectors in measurement step. Then measurement vector is predicted if measurement vector is input.

$$
\begin{gathered}
K(t)=P^{-}(t) H^{t}\left[H P^{-}(t) H^{t}+R(t)\right]^{-1} \\
\hat{s}(t)=\Phi(\Delta t) \hat{s}(t-\Delta t)+K(t)[z(t)-H(t) \Phi(\Delta t) \hat{s}(t-\Delta t)] \\
P(t)=(1-K(t) H(t)) P^{-}(t-\Delta t) \\
K L(t)=P L^{-}(t) H^{t}\left[H P L^{-}(t) H^{t}+R L(t)\right]^{-1} \\
s L^{\wedge}(t)=\Phi(\Delta t) s L^{\wedge^{-}}(t-\Delta t)+ \\
K(t)\left[z L(t)-H(t) \Phi(\Delta t) s L^{\wedge^{-}}(t-\Delta t)\right] \\
P L(t)=(1-K L(t) H(t)) P L^{-}(t-\Delta t)
\end{gathered}
$$

Where, let $\hat{s}^{-}(t)$ denote the state vector of sending bits predicted through state transition in $t$ time and $s L^{\wedge-}(t)$ denote the vector of lost bits respectively. $\hat{s}(t-\Delta t)$ means optimal state vector of sending bits updated in $t-\Delta t$. Let $s L^{\wedge}(t-\Delta t)$ be the optimal state vector of lost or error bits. The covariance matrix about system state of sending bits predicted in $t$ time is expressed by $P^{-}(t)$, which one of lost or error bits predicted is expressed by $P L^{-}(t)$. In addition, $P(t-\Delta t)$ act as a covariance matrix about system state of sending bits in $t-\Delta t$ time. $P L(t-\Delta t)$ is one of lost or damaged bits. In measurement phase, system state is updated after calculating an error between actual measured vectors and measurement vector predicted in state prediction phase. Let $K(t)$ be Kalman gain of sending state in $t$ time, $K L(t)$ be Kalman gain of lost or error bits in $t$ time. $R(t)$ and $R L(t)$ indicate the covariance matrix of sending state and lost or damaged bits respectively about a measurement error. Let $s^{\wedge}(t)$ and $s L^{\wedge}(t)$ be the state vector updated of sending and lost or damaged bits in $t$ time. These processes are shown in formula (13), (14), (15), (16), (17) and (18). 


\section{B. Results of prediction method and discussion}

The applicability of the predicted method is verified by a mathematics experiment. The more detailed analytical settings are illustrated in section III. Let $d_{0}$ denote the close-in reference distance, which is selected according to the sequence order of $15 \mathrm{~m}, 20 \mathrm{~m}, 25 \mathrm{~m}$, and $30 \mathrm{~m}$. Then the channel state information is colleted in a period of distance. Data in successive intervals is collected. We use the above prediction method to obtain predicted bit error rate and compare the characteristic of it with that of original value.

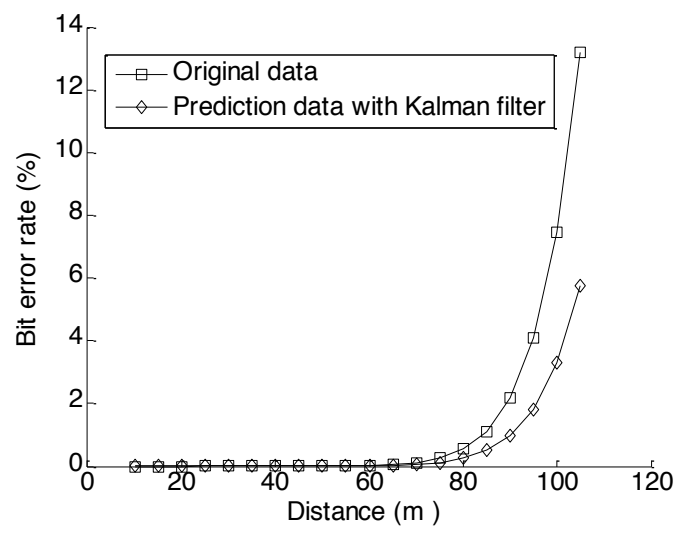

Figure 1 Comparison of original data and prediction data.

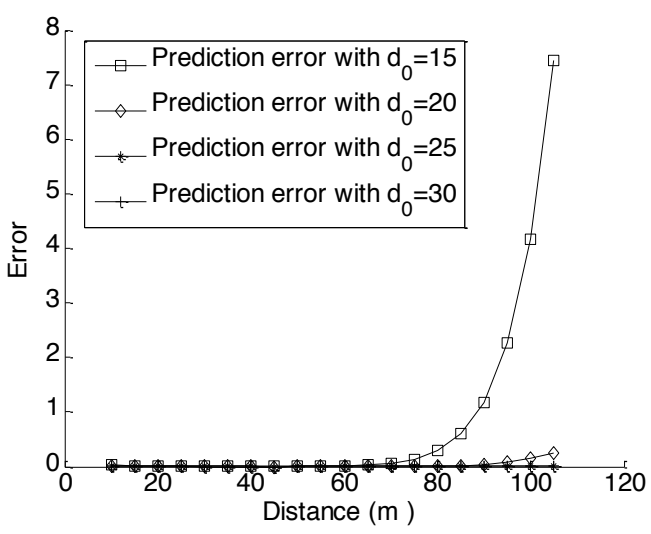

Figure 2 Prediction error with different $d_{0}$

Figure 1 shows the comparison of the original data and the predicted data. Obviously, there is no significant difference between original data and predicted data in the first 15 points, while the difference gradually increases in subsequent 5 points. The chaotic sequence is highly sensitive to the initial value. As distance goes by, the prediction error is getting larger and larger. Besides, it is noticed that the larger the $d_{0}$, the less the prediction error as shown in Figure 2. In order to obtain a relatively accurate data, sensor nodes with large $d_{0}$ should be first used in wireless sensor networks.

\section{SYSTEM MODEL}

In this section, wireless sensor network model based on Mica2 sensor node is presented, an analytic model based on FEC/ARQ is proposed. The variation characteristics of QoS performance with parameters of the hybrid scheme are summarized by mathematical analyses based on the proposed model.

\section{A. Network model}

In the wireless sensor network, Mica2 sensor node is comprised of ATmega128L processor and CC1000 radio module. In order to analyze the variation characteristics of bit error rate hop by hop, the log-distance path loss model is used, which is able to approximate the effects of the signal propagating through the wireless channel. In this model, let $d$ denote the distance between the sender node and receiver node. The signal-to-noise ratio (SNR) with $d$ is given by:

$$
S N R=P_{t}-P\left(d_{0}\right)-10 \beta \lg \left(d / d_{0}\right)-P_{n}
$$

Here, let $\beta$ denote the path loss exponent and assuming that it is set to $2 . d_{0}$ is determined by the physical attribution of the sensor node. $P_{n}$ is the noise power, Mica2 node implements with no coherent FSK modula tion scheme. So the bit error rate of the wireless channel is given by formula (20).

$$
P_{b}=\frac{1}{2} e^{S N R \frac{B_{N}}{2 R_{\text {radio }}}}
$$

Where $B_{N}$ is the noise bandwidth and the data rate of $\mathrm{CC} 1000$ is set to $R_{\text {radio }}$.

The hybrid FEC/ARQ mechanism is proposed, which is shown in Figure 3. In this case, one data packet is encapsulated to $k$ source data units. To these units, $N-k$ redundant units are added, which are decoded by block code FEC, e.g. Reed-Solomon. $N$ denotes the length of the code. If some data units are lost or damaged, they will be dealt with ARQ by $N_{\max }$ retransmission. One packet is decoded if we correctly receive $k$ or more units at the receiver node. We denote $P_{\mathrm{FEC} / \mathrm{ARQ}}$ by the packet error rate with FEC/ARQ, which is expressed by formula (21).

$$
P_{F E C / A R Q}=1-\left(\sum_{k}^{N}\left(\begin{array}{c}
N \\
l
\end{array}\right)\left(1-P_{b}\right)^{l} P_{b}^{N-l}\right)^{l_{D A T A}}
$$

Where, the data packet size $l_{D A T A}$ can be given as the sum of data link layer frame header length and frame check sequence size $\alpha$ plus packet length of ACK $l_{A C K}$. Here, the value of $l_{D A T A}$ and $l_{A C K}$ could be obtained according to the MAC protocol in this network.

Above all, we describe the wireless channel state with four parameters, which are $<N, k, N_{\max }, d_{0}>$.

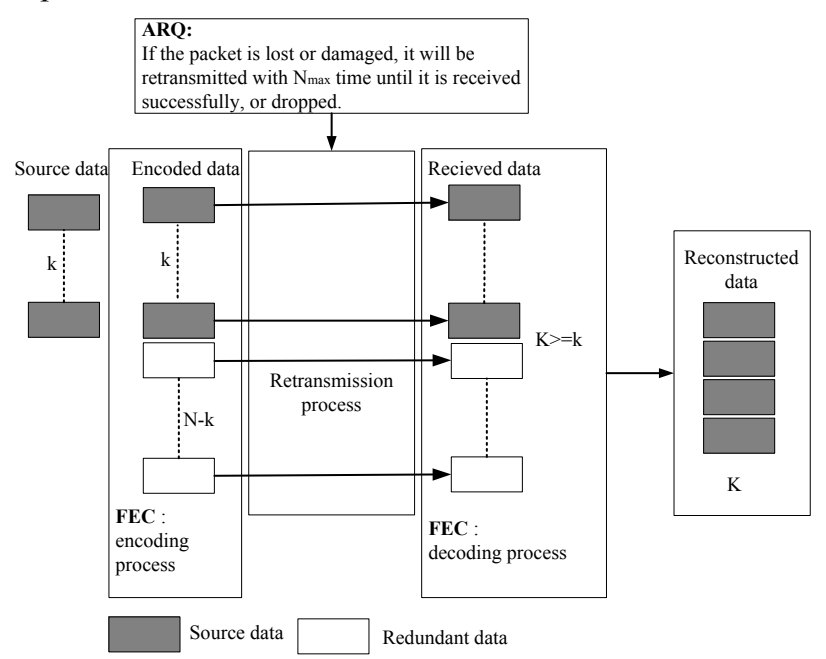

Figure 3 FEC/ARQ model in wireless sensor networks 


\section{B. QoS mathematics model}

In this model, there are four performance metrics, which are listed as follows:

(1) Throughput ratio: the ratio of the payload length to the length of data packets after these packets have been retransmitted $N_{\max }$ time successfully.

(2) Packet error rate: the rate of packets failures reported during the numerical analysis.

(3) Average delay: we only calculate the end to end delay between sender node and receiver node.

(4) Energy efficiency: the number of obtained decodable data packets per unit of energy consumption.

Let $S$ denote the throughput ratio. So we have

$$
S=\frac{l_{\text {payload }}}{l_{D A T A}}\left(1-P_{F E C / A R Q}^{N_{\max }+1}\right)
$$

Here, $l_{\text {payload }}$ is the length of payload. Besides, the packet error rate $P$ after the error control process could be achieved by the following equation.

$$
P=P_{F E C / A R Q}^{N_{\max }+1}
$$

According to the equation (23), we can obtain the solution of average delay, which is shown as equation (24).

$$
T_{F E C / A R Q}=T \frac{1-P_{F E C / A R Q}^{N_{\max }+1}}{1-P_{F E C / A R Q}}
$$

Here, let $T$ denote the round trip time of one packet.

Finally, the energy efficiency $\eta$ is defined as equation (25), which is a suitable metric that could capture the energy and reliability constraints.

$$
\eta=\frac{E_{e f f i}}{E_{\text {total }}}(1-P)
$$

In other words, $\eta$ is the ratio of the energy of payload $E_{\text {effi }}$ to the total energy consumption $E_{\text {total }}$. Hence, the energy efficiency of FEC/ARQ $\eta_{F E C / A R Q}$ is given as:

$$
\eta_{\mathrm{FEC} / \mathrm{ARQ}}=\frac{\mathrm{E}_{\text {effi }}^{\text {ARQ }}}{E_{\text {total }}^{A R Q}}\left(1-P_{\mathrm{FEC} / \mathrm{ARQ}}\right)=\frac{l_{\text {payload }}}{l_{\text {DATA }}}\left(1-P_{\mathrm{FEC} / \mathrm{ARQ}}\right)
$$

\section{Results and discussion}

In this subsection, the influence of channel state with four performance metrics is studied at first. Then, the characteristic of channel state based on FEC/ARQ is discussed and the uniqueness of threshold value of bit error rate is proved finally.

On the one hand, the impact of $N, k, N_{\max }$ and $d_{0}$ is illustrated in Figure 4, 5, 6 and 7, which show four performance metrics as a function of channel state. The energy efficiency and throughput ratio increase quick when the value of $N, k, N_{\max }$ and $d_{0}$ is raised gradually as shown in Figure 4 and 7 . In contrast, the trend of packet error rate and average delay descend fast along with the value of four parameters as shown in Figure 5 and 6 . However, the delay would be greater when the value of $N_{\max }$ is larger.

On the other hand, the change trend of four performance metrics as a function of $P_{b}$ is shown as Figure 8. It was obvious that the performance of FEC/ARQ is maintained gradually perfect with decreasing of $P_{b}$. Specially, the throughput ratio and energy efficiency of this scheme decrease to zero rapidly, meanwhile, the packet error rate and average delay increase fast when the $P_{b}$ is greater than 0.0003 , when $N=20, k=6, N_{\max }=2$ and $d_{0}=20$. Therefore, $P_{b}$ has a constant value, which is 0.0003 for this scheme. There is apparently a $P_{b}$ threshold value. Likewise, the $P_{b}$ threshold value is 0.0004 with $N=20, k=6, N_{\max }=3$ and $d_{0}=20$, as well as the threshold is 0.00055 with $N=20$, $k=6, N_{\max }=2$ and $d_{0}=25$. Therefore, different channel state has always one constant $P_{b}$ threshold value. The optimal relay node could be chosen according to this conclusion.
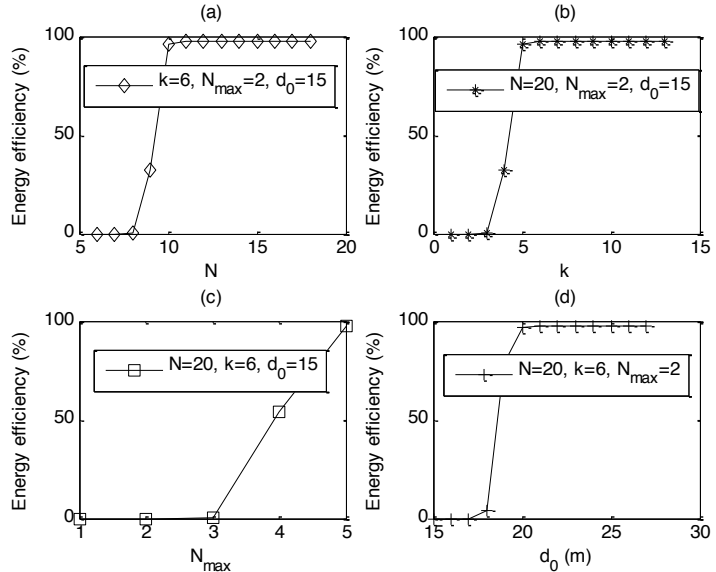

Figure 4 Energy efficiency analyses with channel state. (a) $N$, (b) $k$, (c)
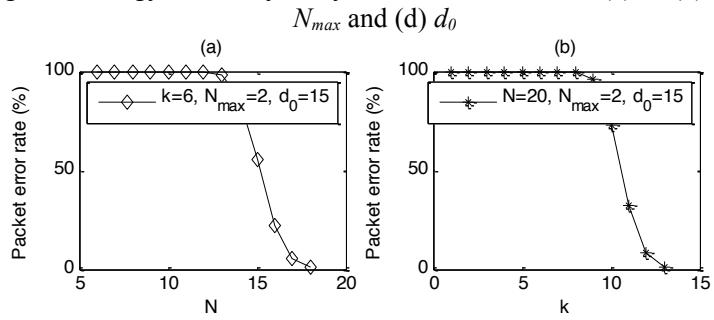

(c)
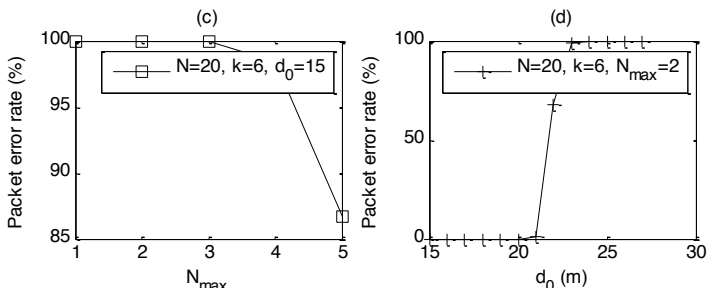

Figure 5 Packet error rate analyses with channel state. (a) $N$, (b) $k$, (c)
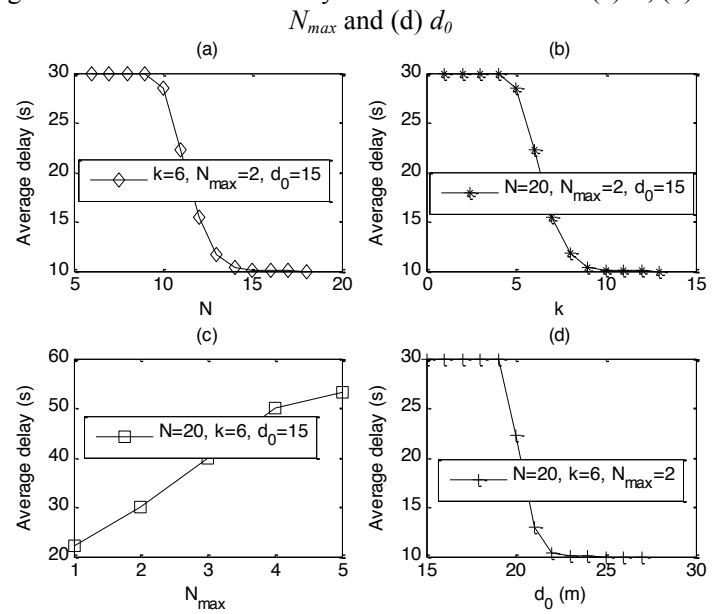

Figure 6 Average delay analyses with channel state. (a) $N$, (b) $k$, (c) $N_{\max }$ and (d) $d_{0}$ 

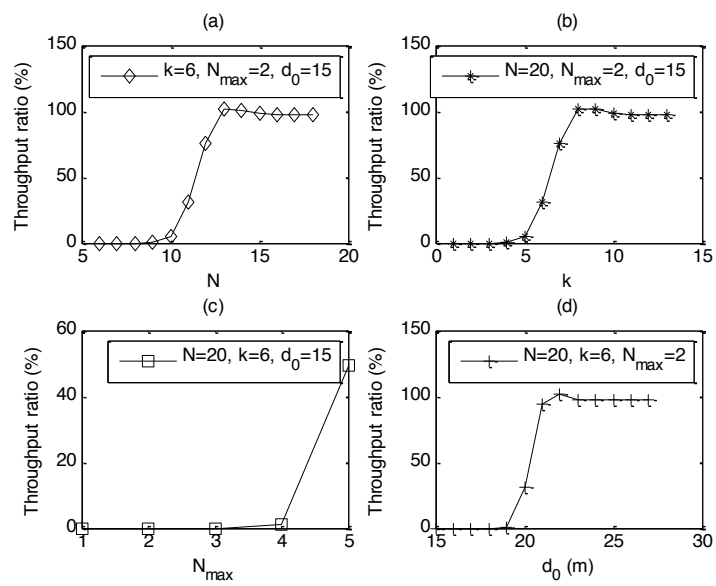

Figure 7 Throughput ratio analyses with channel state. (a) $N$, (b) $k$, (c) $N_{\max }$ and (d) $d_{0}$
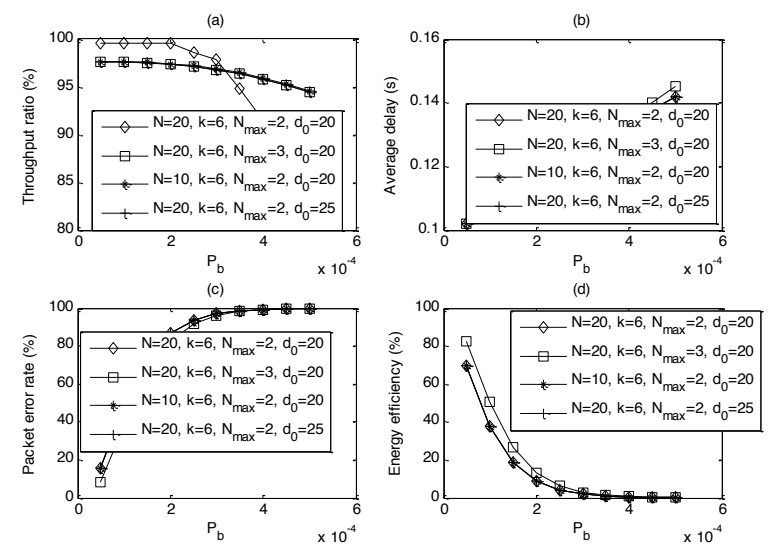

Figure 8 Performance analyses with $P_{b}$. (a) throughput ratio, (b) average delay, (c) packet error rate and (d) energy efficiency

\section{Channel AWARE COOPERATIVE FEC/ARQ}

In this section, we present the basic idea of the Channel Aware Cooperative FEC/ARQ (CAC-FEC/ARQ) and its implementation in wireless sensor networks in detail, which is illustrated as follows:

Procedure of CAC-FEC/ARQ at sender node as shown in Figure 9:

Step (1) Carry out the Kalman filter prediction scheme. The bit error rate of candidate node is forecasted on the basis of the statistics of listening channel.

Step (2) $N, k, N_{\max }$ and $d_{0}$ are specified on account of QoS analytical model. Moreover, the value of $P_{b}$ is obtained based on the variations characteristics of QoS performance.

Step (3) Relay selection mechanism based on $P_{b}$ is implemented when channel state information is known.

Step (4) According the channel state parameter, FEC scheme is executed for sending data packets. If ACK packet is received, new data packets are sent and retransmission time-out is used for each packet at the same time.

Step (5) When the timer matures or NACK packet is received, the ARQ scheme is started to retransmit the lost or damaged packets according to the channel state.

Relay selection procedure:

Step (6) Select the optimal relay node from candidate nodes based on $P_{b}$ threshold.
Step (7) Steps (3), (4) and (5) are implemented repeatedly according to the order of sequence until the data packet is received successfully or discarded actively.

Procedure of CAC-FEC/ARQ at receiver node:

Step (8) Checksum testing and FEC decoding are implemented.

Step (9) If the examination and result is right, the data packet is accepted and ACK packet is sent simultaneously; otherwise, it is dropped and NACK packet is sent immediately.

Step (10) Accurate data packets are delivered to the upper layer.

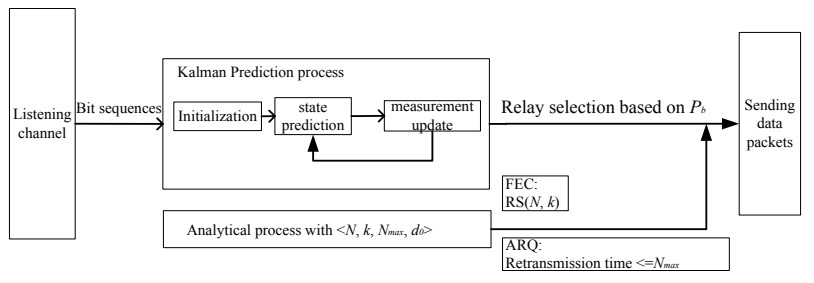

Figure 9 Process of CAC-FEC/ARQ at sender node

\section{Performance evaluation}

In this work, we simulate, analyze and evaluate the performance of CAC-FEC/ARQ, compared with traditional FEC/ARQ, Cooperative FEC (CFEC) and Cooperative ARQ (CARQ) through two group experiments. The experimental data is the average value after 100 time simulation and mathematical analysis.

\section{A. Simulation environment}

In our mathematical analysis, 30 mobile sensor nodes move in a $1,000 \mathrm{~m} \times 800 \mathrm{~m}$ rectangular region. All nodes cover the same transmission range of $200 \mathrm{~m}$. The mobility model is the random way point model. Each node moves towards the destination at a speed uniformly chosen between the minimal speed and maximal speed. Here, the minimal speed is $0 \mathrm{~m} / \mathrm{s}$ and maximal speed is $20 \mathrm{~m} / \mathrm{s}$. The pause time is $0 \mathrm{~s}$. The Mathematical environment is illustrated in Table I.

In our NS simulation experiment, a medium quality MPEG4 video clip from the movie StarWars IV is used, which is consisted of 89,998 video frames (I-frame, Pframe and B-frame). The group of picture is comprised of IBBPBBPBBPBB. The video frame rate is 30 video frames per second. The mean bit rate is $77.72 \mathrm{Kbps}$ and the peak value is $938 \mathrm{Kbps}$. The more detailed simulation parameter settings are illustrated in Table II.

TABLE I.

PARAMETER SETTINGS OF MATHEMATICAL MODEL

\begin{tabular}{cc}
\hline Parameters & Value \\
\hline Packet size & 1500 bytes \\
ARQ ACK Type & Selective \\
$T$ & $0.1 \mathrm{~s}$ \\
$\alpha$ & 11 bytes \\
$l_{A C K}$ & 7 bytes \\
$B_{N}$ & $30 \mathrm{kHz}$ \\
$R_{\text {radio }}$ & $38.4 \mathrm{kBaud}$ \\
MAC protocol & IEEE 802.15 .4 \\
\hline
\end{tabular}


TABLE II.

PARAMETERS SETTINGS OF SIMULATION

\begin{tabular}{ll}
\hline \multicolumn{1}{c}{ Parameters } & \multicolumn{1}{c}{ Value } \\
\hline Simulation time & $120 \mathrm{sec}$ \\
Topology size & $800 \mathrm{~m}^{*} 800 \mathrm{~m}$ \\
MAC protocol & IEEE802.15.4 \\
Route protocol & DSR \\
Motion model & Random waypoint \\
Traffic model & Spread randomly \\
CBR packet size & 512 bytes \\
Pause time of mobile nodes & 0 sec \\
Number of sessions & 1 \\
\hline
\end{tabular}

\section{B. imulation Results and evaluation}

Figure 10 shows four performance metrics as a function of $P_{b}$ in experiment 1 . We can observe tremendous improvement of performance using CACFEC/ARQ, as compared with the traditional hybrid mechanism. Even at remote communication, the quality using CAC-FEC/ARQ maintains perfect performance, while the performance of the traditional scheme is close to bad condition gradually. First of all, the proposed mechanism based on cooperative communication with Kalman filter prediction improves the energy efficiency, the channel aware error control strategy minimizes the average delay next, the network throughput utilization increased finally.
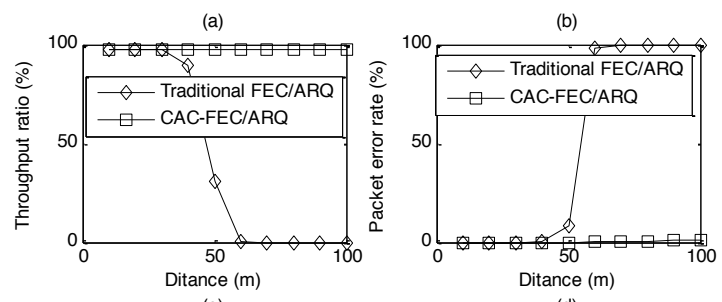

(c)
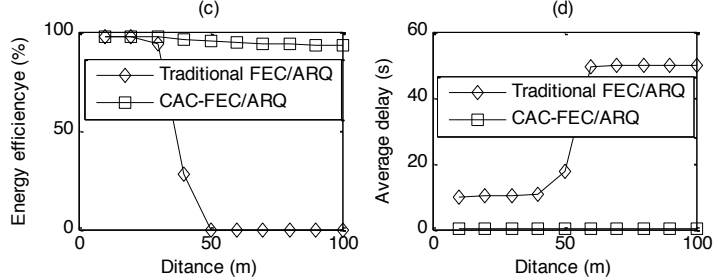

Figure 10 Performance with varying distance. (a) Throughput ratio, (b) packet error rate, (c) Energy efficiency and (d) Average delay

Four performance metrics as a function of different error control schemes in experiment 2 are shown in Figure 11. The system throughput ratio is appeared in Figure 11 (a). Obviously, the CAC-FEC/ARQ always achieves better performance than the other two cooperative error control mechanisms. The throughput gain profits from the enhancement of hop-by-hop throughput on the basis of channel aware relay selection.

Figures 11 (b) presents the packet error rate in different mechanisms. As shown in the result, CAC-FEC/ARQ provides the best reliability of video transmission, next is CARQ, followed by CFEC. When CAC-FEC/ARQ is used, the packet error rate has little fluctuation, and the average is least. Figure 11 (c) shows the energy efficiency of different mechanisms. Clearly, the improvement of data forwarding gain and channel aware error control is able to decrease greatly energy consumption. Comparing with the other two schemes, CAC-FEC/ARQ acquires superior improvement in energy utilization efficiency.

The average delay of CAC-FEC/ARQ achieves a tremendous improvement, compared with one of CFEC and CARQ, which is shown in Figure 11 (d). The channel aware relay selection strategy based on Kalman filter prediction improves the utilization of frequency spectrum; as a result, propagation path is optimized.

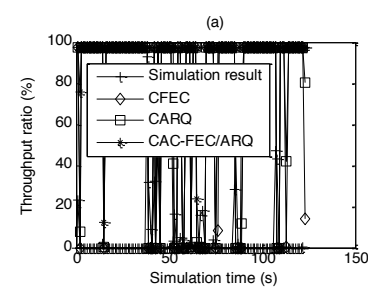

(c)
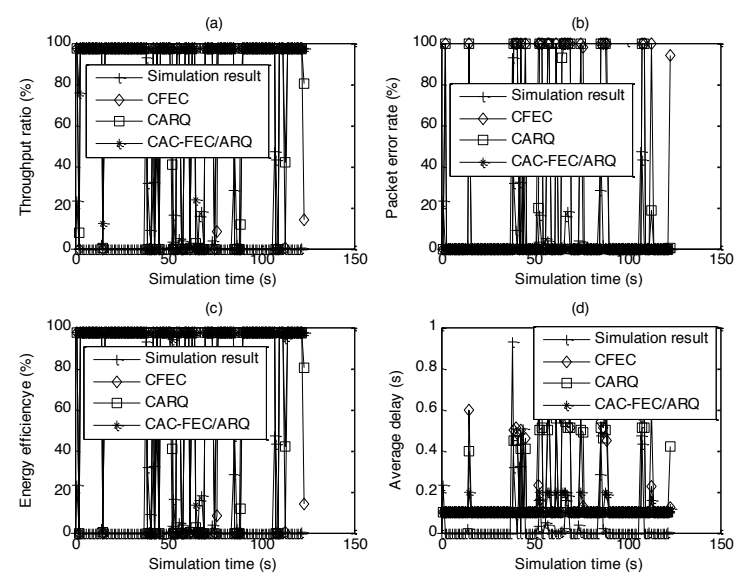

Simulation time (s)
Figure 11: Performance with simulation time. (a) Throughput ratio, (b) packet error rate, (c) Energy efficiency and (d) Average delay

\section{CONCLUSIONS}

In this paper, a channel aware cooperative FEC/ARQ mechanism was proposed for supporting the wireless application in sensor networks, which is based on Kalman filter prediction method. A relay selection scheme was proposed after analyzing the performance of FEC/ARQ with dynamic channel state. We apply the cooperative FEC/ARQ mechanism to decrease the average delay and packet error rate, as well as improvement of throughput and energy consumption. Our investigations in both analytic and simulation approaches demonstrate that the proposed mechanism provides a significant enhancement in the reliability and real-time performance of wireless communication, as well as lifetime maximization of sensor network, with adapting to the fluctuation of the wireless channel state dynamically.

\section{REFERENCES}

[1] F. Akyildiz, W. Su, Y. S. subramaniam, and E. Cayirci, A Survey on sensor networks, IEEE Communication Magazine, Vol. 40 no.8, pp. 102-114, 2002. http://dx.doi.org/10.1109/MCOM. 2002.1024422

[2] F. Xia, QoS Challenges and Opportunities in Wireless Sensor/Actuator Networks, Sensors, vol. no. 8, pp. 1099-1110, 2008.

[3] D. Djenouri, I. Balasingham, Traffic-Differentiation-Based Modular QoS Localized Routing for Wireless Sensor Networks, IEEE Transactions on Mobile Computing, , Vol. 10, Issue 6, pp: 797-809, 2012.

[4] Y. Jin, G. W. Bai, and P. Zhang, et al, Performance Evaluation of a hybrid FEC/ARQ for Wireless Media Streaming, Proceedings of IEEE International Conference on Circuits and Systems for Communications, pp. 90-94, Shanghai, China, 2008.

[5] M. Tacca, P. Monti and A. Fumagalli, Cooperative and Reliable ARQ Protocols for Energy Harvesting Wireless Sensor Nodes, 
IEEE TRANSACTIONS ON WIRELESS COMMUNICATIONS, vol. 6, no. 7, pp: 2519-2529, 2007. http://dx.doi.org/10.1109/TWC. 2007.05878

[6] C. H. Shih, Enhancing Packet-level Forward Error Correction for Streaming Video in Wireless Networks, International Journal of Computer Science Issues, Vol. 9, no. 5, no. 2, pp: 146-155, 2012.

[7] Y. Jin and G. W. Bai, A Cooperative FEC based on the model of GM $(1,1)$ and IPv6 for Wireless Multimedia Sensor Networks, Journal of Convergence Information Technology, Vol. 7, No. 18, pp. 230-239, 2012. http://dx.doi.org/10.4156/jcit.vol7.issue18.28

[8] M. Mardani, J. S. Harsini and F. Lahouti, et al, Link adaptive and QoS provisioning cooperative ARQ - Application to relay assisted land mobile satellite communication, IEEE Transaction on vehicular technology, vol. 60, no. 7, pp: 3192-3206, 2011. http://dx.doi.org/10.1109/TVT.2011.2151261

[9] Natarajan Meghanathan, and Gordon W. Skelton. Risk Notification Message Dissemination Protocol for Energy Efficient Broadcast in Vehicular Ad hoc Networks[J]. IAENG International Journal of Computer Science, Vol. 37, No. 1, 2010.

[10] M. ELHAWARY, Z. J. HAAS, Energy-Efficient Protocol for Cooperative Networks [J]. IEEE/ACM Transactions on Networking. Vol. 19, no. 2, pp: 561-574, 2011. http://dx.doi.org/10.1109/TNET.2010.2089803

[11] S. R. Cho, C. Wan and K. B. Huang, QoS Provisioning Relay Selection in Random Relay Networks, IEEE Transactions on Vehicular Technology, vol. 60, no. 6, pp. 2680-2689, 2011. http://dx.doi.org/10.1109/TVT.2011.2153220

[12] G. Amarasuriya, M. Ardakani and C. Tellambura, OutputThreshold Multiple-Relay- Selection Scheme for Cooperative
Wireless Networks, IEEE Transactions on Vehicular Technology, vol. 59, no. 6, pp. 3091-3097, 2010. http://dx.doi.org/10.1109/ TVT.2010.2048767

[13] M. Y. Huanga and S. Deyb, Stability of Kalman filtering with Markovian packet losses, Automatica, vol. 43, pp. 598 - 607, 2007. http://dx.doi.org/10.1016/j.automatica.2006.10.023

[14] W. Kim, H. J. Jang and G. Y. Kim, Transmission Rate Prediction of VBR Motion Image Using the Kalman Filter, Proceedings of ICCSA, LNCS 3981pp. 106-113, 2006.

\section{AUTHORS}

Yong Jin received a master of engineering (2009) in Computer Science and Technology from the Nanjing University of Technology. Since 2009, he has been working at the Changshu Institute of Technology, as a lecturer in School of Computer Science and Engineering, Changshu 215500, China. His research interests are in mobile ad hoc networks, multimedia communication, cooperative communication, wireless sensor networks, QoS, performance evaluation, error control, etc. (e-mail: jinyong@cslg.cn).

Submitted 04 September 2013. Published as re-submitted by the authors 23 January 2014. 